\title{
Papel de la resistencia a la insulina y el hiperinsulinismo en el desarrollo de síndromes de androgenización
}

\author{
Dres. Bernardo Reyes Leal*, Amanda Páez Talero**
}

RESUMEN: En investigaciones anteriores se ha demostrado una correlación estadística entre el índice de masa corporal (BMI) y el grado de hiperandrogenismo, éste estudio pretende determinar si el exceso de insulina estaba presente en esta clase de pacientes. Para evitar el sesgo que pueda representar el hiperinsulinismo y obesidad "perse" los pacientes fueron subdivididos en cuatro grupos:

Grupo I controles normales - Sin obesidad o hirsutismos

Grupo II con obesidad e hirsutismo

Grupo III con obesidad sin hirsutismo

Grupo IV sin obesidad con hirsutismo

Se realiza examen físico cuidadoso y los siguientes examenes de laboratorio: Glicemia e insulina antes, a los $30,60,120$ y 180 mínutos después de glucosa Oral; Dosificación de testosterona libre y total.

Las Areas de curva de glucosa e insulina fueron obtenidas y el índice de resistencia a la insulina fue calculado. El hiperinsulinismo y la resistencia a la insulina estuvo presente en los tres grupos de Pacientes. Sin embargo, el índice de resistencia a la insulina fue más alto en el grupo II, 9.3 que en los otros dos grupos. 6.2 en el grupo III y 3.1 en el grupo IV. Es interesante destacar que dos alteraciones aparecen relacionadas con un cierto grado de resistencia a la insulina; para la obesidad 6.2 y para el hirsutismo 3.1. La asociación de estas dos condiciones da un índice de $9.3(6.2+3.1)$.

PALABRAS CLAVES: Hiperinsulinemia, resistencia a la insulina, síndrome de androgenización.

SUMMARY. Based on data obtained in a previous investigation which showed a statistical correlation between the body mass index (BMI) and the degree of hyperandrogenism, this study was designed in order to see if an excess of insulin was present in this kind of patients. In order to avoid the biass that may represent the hyperinsulinism of obesity per se, the subjects were subdivided in four groups:

GROUP I - Normal controls without obesity or hirsutism.

GROUP II - Subjects with both obesity and hirsutism.

GROUP III - Subjects with obesity, without hirsutism.

GROUP IV - Subjects without obesity, with hirsutism.

Carefull physical examination and the following laboratory test were performed: blood glucose and insulin before and 30 , 60,120 and 180 minutes after oral glucose; total and free testosterone.

The areas under the curve of glucose and insuline were obtained, and an index of insulin resistance calculated. Hyperinsulinism and insulin resistance were present in the three groups of patients. However the index of insulin resistance was much higher in group II (obesity plus hirsutism), 9.3, than in the other two groups. 6.2 in the group III, obese without hirsutism, and 3.i in group IV, hirsutes non obese. It is interesting to underline that the two disturbances appear as if each one was related with a certain degree of insulin resistance; obesity accounting for 6.2 and hirsutism for 3.1. The association of the two conditions gave an index of $9.3(6.2-3.1)$.

KEY WORDS: Hyperinsulinism, insulin resistance, androgenism syndrome

\section{Introducción}

Desde hace varios años la Sección de Endocrinología del Hospital San Juan de Dios (H.S.J.D.) ha prestado particular atención a los fenómenos de androgenización en la mujer, motivo de consulta frecuente en nuestra práctica diaria. La razón de este interés está relacionada con el hecho de que un exceso de insulina, evento característico de la diabetes

\footnotetext{
* Profesor Titular y Emérito, Sección de Endocrinología, Dpto. de Medicina Interna, Facultad de Medicina, Universidad Nacional de Colombia.

** Residente IV Año Endocrinología, Unidad de Endocrinología, Facultad de Medicina, Universidad Nacional de Colombia.
}

mellitus tipo II (1) y un hallazgo constante en los sujetos obesos (2), parece ser el elemento esencial en la génesis de esta patología; existe al respecto a nivel mundial una abundante y reciente bibliografía (3-9).

En la última década la resistencia a la insulina y su consecuencia, el hiperinsulinismo, han dejado de ser considerados como factores relacionados únicamente con alteraciones del metabolismo de carbohidratos, y se piensa que tienen un papel etiológico importante en cuadros patológicos considerados hasta ahora como "esenciales"; se postula por ejemplo, una relación directa entre la hiperinsulinemia y la hipertensión arterial $(10,11)$, la hiperlipidemia $(11)$, la hi- 
peruricemia (12) y en lo que concierne a nuestro trabajo, los fenómenos de androgenización en la mujer (13-16).

Numerosas descripciones desde el síndrome de AchadThiers o "Diabetes de la Mujer Barbada" en 1921 (17), el síndrome de Stein-Leventhal o Síndrome de Ovario Poliquístico en 1935 (18) y los síndromes de Hiperandrogenismo, Resistencia a la Insulina y Acantosis Nigricans HAIR-AN (4), indicaban la relación entre una elevada concentración de insulina y un exceso de andrógenos evidenciado clínicamente en el hirsutismo, las irregularidades menstruales y la infertilidad $(13,14)$. El origen de esta sobreproducción andrógena se ha establecido en el ovario mediante cateterismo de vena suprarrenal y vena ovárica (19) y por esto, numerosos estudios se han dirigido hacia las células ováricas para establecer la acción de la insulina y de factores de crecimiento tipo IGF I sobre ellas. El primer paso consistió en demostrar la presencia de receptores para estas hormonas en el ovario y es así como Rein y Schomberg identifican receptores para la insulina (20) y Veldhuis y col, para IGF I (21), en las células de la granulosa de ovarios porcinos. Los posibles mecanismos mediante los cuales la insulina y los factores de crecimiento estimulan la esteroidogénesis ovárica, son indirectos, incrementando el transporte de glucosa y aminoácidos al interior de la célula y la síntesis de proteínas, causando proliferación celular de la granulosa, en forma proporcional a la concentración de insulina $(20,22)$; de otra parte se ha comprobado una acción directa de la insulina modificando la actividad de aromatasa (23), actuando en forma sinérgica con la LH o FSH y modulando los receptores ováricos para la LH $(20,24,25)$ : acciones directas sobre la esteroidogénesis que culminan en una elevada secreción de estrógenos y progesterona por las células de la granulosa y de androstenediona y testosterona por la teca y el estroma (20-25).

Nosotros, en acuerdo con estas afirmaciones y tomando como base el estudio previo de la sección, en el cual se encontró una correlación positiva entre el índice de masa corporal (IMC) y los niveles de testosterona total (TT) en mujeres hirsutas (26), nos propusimos adelantar en la investigación del posible papel de la insulina en el desarrollo de síndromes de androgenización en la mujer.

El planteamiento inicial postuló la obesidad, que se acompaña siempre de hiperinsulinismo, como el evento que desencadenaría todas las alteraciones en la secreción de andrógenos por el ovario; sin embargo, nosotros encontrábamos en nuestra consulta situaciones contradictorias; por ejemplo, observábamos mujeres hiperandrogénicas de peso normal. ¿Cómo explicaríamos entonces en ellas el evento fisiopatológico desencadenante de su androgenización? De otra parte encontrábamos mujeres obesas, algunas con Acantosis Nigricans, que no presentaban ningún síntoma de hiperfunción androgénica. ¿Cómo explicaríamos la ausencia de androgenización de estas mujeres necesariamente hiperinsulinémicas? ¿Serían estos eventos el resultado de una cadena de alteraciones predecible o están involucrados otros mecanismos propios a cada grupo de mujeres de los cuales las caracterizan? Si planteamos una mayor acción de la insulina sobre el ovario, ¿cómo explicar el exceso de acción de una hormona sobre sus receptores, en un estado de resistencia periférica a la misma?

Tratando de resolver estos interrogantes, nosotros preten- demos determinar los niveles de glicemia e insulinemia, tanto basales como estimulados por la glucosa y de andrógenos, en cuatro grupos de mujeres diferenciados así: Grupo I: Mujeres controles normales (C).

Grupo II: Mujeres obesas hiperandrogénicas (OA). Grupo III: Mujeres obesas sin hiperandrogenismo (O). Grupo IV: Mujeres hiperandrogénicas de peso normal (A).

Queremos establecer las diferencias entre las variables examinadas, para determinar el impacto de la obesidad y la hiperinsulinemia sobre la función gonadal en estas mujeres.

\section{Sujetos y métodos}

Población. Se incluyeron en el estudio 65 mujeres en edad reproductiva (16-43 años), con un promedio de 26.1 años, 15 de las cuales consideradas como controles (C), Grupo I, fueron seleccionadas entre estudiantes de medicina; tenían un IMC menor de 25 , una historia menstrual regular y ningún signo de hiperfunción androgénica.

Las 50 restantes se recolectaron de las pacientes que consultan a la Sección de Endocrinología del H.S.J.D., por presentar hirsutismo, acompañado o no de obesidad; o por obesidad sin hirsutismo, entre marzo 1o. de 1987 y agosto 30 de 1988. Tales mujeres fueron distribuidas así:

Grupo II: Pacientes obesas con signos de hiperandrogenismo (OA) $\mathrm{n}=20$.

Grupo III: Pacientes obesas sin signos de hiperandrogenismo (O) $\mathrm{n}=15$.

Grupo IV: Pacientes no obesas con signos de hiperandrogenismo (A) $n=15$.

Fueron consideradas como obesas aquellas que tenían un IMC mayor de 25 y como hiperandrogénicas las que tenían un índice de hirsutismo (IH) de 5 a 20/20, aplicando la calificación de Moncada Lorenzo (27). Se consideraron como signos accesorios de androgenización la presencia de acné y la historia de oligomenorrea o amenorrea. A cada mujer le fueron investigados sus antecedentes patológicos personales o familiares, haciendo énfasis en aquellos relacionados con la diabetes mellitus tipo II en parientes en primer grado de consanguinidad. Se estableció que no estuvieran tomando ninguna medicación por lo menos tres meses antes de la realización del estudio. Dentro de su examen físico se incluyó la estimación de su IMC, IH y la presencia de acné y/o Acantosis Nigricans.

Las características clínicas de las pacientes de cada uno de los cuatro grupos se ilustran en las Tablas $1 \mathrm{a}, 1 \mathrm{~b}, 1 \mathrm{c}$, 1d, y se resumen en la Tabla le.

Todas las mujeres fueron informadas del procedimiento y dieron su aprobación para ingresar al estudio.

Diseño del estudio. El estudio fue realizado en el laboratorio de la Sección de Endocrinología del H.S.J.D., por el personal a cargo. Las determinaciones de glucosa fueron realizadas inmediatamente y los sueros congelados $\mathrm{a}-20^{\circ} \mathrm{C}$ para la determinación posterior de insulina y andrógenos. Ninguna de las muestras para medida de insulina permaneció congelada por un período de tiempo superior a dos meses; por el contrario algunos de los sueros para determinar andrógenos, permanecieron almacenados por un tiempo superior.

Después de instruir a las pacientes para que llevaran una dieta libre durante tres días antes del estudio y de permanecer 
Tabla 1a

PRESENTACION CLINICA DE LAS MUJERES DEL GRUPO I $n=15$ CONTROLES $(C)$

\begin{tabular}{|c|c|c|c|c|c|c|c|}
\hline PTE.No. & EDAD & $\begin{array}{c}\text { ANTEC. } \\
\text { DMII }\end{array}$ & $\begin{array}{l}\text { OLIGOAM. } \\
\text { AMENORREA }\end{array}$ & IMC & IH & $\overline{A N}$ & ACNE \\
\hline 1 & 26 & + & - & 22.7 & $0 / 20$ & - & - \\
\hline 2 & 21 & - & - & 18.7 & $0 / 20$ & - & - \\
\hline 3 & 22 & - & - & 19.5 & $0 / 20$ & - & - \\
\hline 4 & 25 & - & - & 21.3 & $0 / 20$ & - & - \\
\hline 5 & 23 & + & - & 21.3 & $0 / 20$ & - & - \\
\hline 6 & 22 & - & - & 21.4 & $0 / 20$ & - & - \\
\hline 7 & 21 & - & - & 19.0 & $0 / 20$ & - & - \\
\hline 8 & 28 & + & - & 24.0 & $0 / 20$ & - & - \\
\hline 9 & 28 & - & - & 20.6 & $0 / 20$ & - & - \\
\hline 10 & 26 & - & - & 19.1 & $0 / 20$ & - & - \\
\hline 11 & 23 & - & - & 20.0 & $0 / 20$ & - & - \\
\hline 12 & 20 & - & - & 20.8 & $0 / 20$ & - & - \\
\hline 13 & 23 & - & - & 18.5 & $0 / 20$ & - & - \\
\hline 14 & 22 & + & - & 19.3 & $0 / 20$ & - & - \\
\hline 15 & 28 & + & - & 22.0 & $0 / 20$ & - & - \\
\hline $\bar{X}$ & 23.8 & & & 20.5 & & & \\
\hline RANGO & $20-28$ & & & $18.5-24.0$ & & & \\
\hline SEM & \pm 0.7 & & & \pm 0.4 & & & \\
\hline
\end{tabular}

Tabla $1 b$

PRESENTACION CLINICA DE LAS MUJERES DEL GRUPO II $n=20$ OBESAS HIPERANDROGENICAS $[\mathrm{OA})$

\begin{tabular}{|c|c|c|c|c|c|c|c|}
\hline PTE. No. & EDAD & $\begin{array}{l}\text { NTEC. } \\
\text { DM II }\end{array}$ & $\begin{array}{l}\text { OLIGOAM. } \\
\text { AMENORREA }\end{array}$ & IMC & IH & AN & ACNE \\
\hline 1 & 30 & + & + & 28.9 & $5 / 20$ & + & + \\
\hline 2 & 38 & + & + & 25.4 & $15 / 20$ & + & - \\
\hline 3 & 38 & $+*$ & + & 40.0 & $14 / 20$ & + & - \\
\hline 4 & 35 & - & - & 41.3 & $9 / 20$ & + & + \\
\hline 5 & 36 & + & + & 27.3 & $11 / 20$ & + & + \\
\hline 6 & 30 & + & + & 30.2 & $15 / 20$ & + & - \\
\hline 7 & 21 & - & - & 32.9 & $8 / 20$ & + & + \\
\hline 8 & 19 & $+*$ & + & 27.3 & $10 / 20$ & + & + \\
\hline 9 & 31 & $+*$ & - & 35.5 & $5 / 20$ & + & - \\
\hline 10 & 26 & - & + & 25.6 & $9 / 20$ & - & + \\
\hline 11 & 23 & + & - & 35.7 & $14 / 20$ & - & + \\
\hline 12 & 28 & - & + & 25.9 & $5 / 20$ & - & - \\
\hline 13 & 30 & + & + & 32.0 & $5 / 20$ & - & + \\
\hline 14 & 32 & + & + & 30.9 & $16 / 20$ & - & + \\
\hline 15 & 25 & + & + & 25.9 & $13 / 20$ & - & - \\
\hline 16 & 26 & + & + & 29.0 & $7 / 20$ & - & - \\
\hline 17 & 27 & + & + & 27.7 & $5 / 20$ & - & - \\
\hline 18 & 25 & - & + & 34.5 & $6 / 20$ & - & - \\
\hline 19 & 18 & $+*$ & + & 25.3 & $9 / 20$ & - & + \\
\hline 20 & 26 & - & + & 28.8 & $10 / 20$ & - & + \\
\hline $\bar{X}$ & 28.2 & & & 30.5 & $12 / 20$ & & \\
\hline RANGO & $18-38 \mathrm{~A}$ & & & $25.3-41.3$ & & & \\
\hline SEM & \pm 1.2 & & & \pm 1.0 & & & \\
\hline
\end{tabular}

* Antecedente bilateral de DM II

en ayuno desde la noche anterior, se les practicó un test de tolerancia con 100 grs de glucosa oral y se tomaron muestras para la determinación de glicemia e insulinemia a los 0,30 , 60,120 y 180 minutos. De la muestra basal fueron separados y congelados sueros para determinación posterior de TT y TL. Se instruyó también a las pacientes para la realización de una ecografía pélvica en la Sección de Imágenes Diagnósticas de nuestra institución.

Laboratorio. Las determinaciones de glucosa fueron realizadas por el método de glucosa oxidasa-peroxidasa-PAP No. 3395 de la casa Merck. Los niveles de insulina, testosterona total y testosterona libre fueron determinados con los respectivos kits comerciales para radioinmunoanálisis "Coat-A-Count" de DPC.

Análisis de los datos. Los datos obtenidos en las curvas de glicemia e insulinemia fueron analizados determinando el área bajo la respectiva curva; para ello se utilizó la aplicación del método trapezoidal, no a partir de la cifra basal, sino a partir de cero, puesto que encontramos valores a las tres horas inferiores a los basales; se utilizó por lo tanto la siguiente fórmula:

$\frac{0+30 \times 0.5}{2} \frac{+30+60 \times 0.5}{2} \frac{+60+120 \times 1}{2} \frac{+120+180 \times 1}{2}$

Los resultados obtenidos fueron procesados en el Centro de Cómputo de la Universidad Nacional, en un computador IBM utilizando el programa estadístico SAS (28). Los resultados son expresados en promedios más o menos el error

Tabla 1c

PRESENTACION CLINICA DE LAS MUJERES DEL GRUPO III $\mathbf{n}=15$ OBESAS NO HIPERANDROGENICAS (O)

\begin{tabular}{|c|c|c|c|c|c|c|c|}
\hline PTE.No. & EDAD & $\begin{array}{l}\text { ANTEC. } \\
\text { DM II }\end{array}$ & $\begin{array}{l}\text { OLIGOAM. } 0 \\
\text { AMENORREA }\end{array}$ & IMC & IH & $\overline{A N}$ & ACNE \\
\hline 1 & 34 & + & - & 45.6 & $0 / 20$ & + & - \\
\hline 2 & 26 & - & - & 45.0 & $0 / 20$ & + & - \\
\hline 3 & 16 & $+*$ & - & 32.9 & $0 / 20$ & + & - \\
\hline 4 & 40 & + & - & 31.8 & $0 / 20$ & + & - \\
\hline 5 & 25 & + & - & 42.3 & $0 / 20$ & + & - \\
\hline 6 & 28 & + & - & 35.8 & $0 / 20$ & - & - \\
\hline 7 & 38 & + & - & 36.6 & $0 / 20$ & - & - \\
\hline 8 & 27 & + & - & 34.2 & $0 / 20$ & - & - \\
\hline 9 & 34 & + & - & 34.4 & $0 / 20$ & - & - \\
\hline 10 & 17 & $+*$ & - & 29.2 & $0 / 20$ & - & - \\
\hline 11 & 28 & + & - & 26.6 & $0 / 20$ & - & - \\
\hline 12 & 28 & - & + & 44.5 & $0 / 20$ & - & - \\
\hline 13 & 21 & + & - & 27.7 & $0 / 20$ & - & - \\
\hline 14 & 43 & + & + & 40.1 & $0 / 20$ & - & - \\
\hline 15 & 20 & - & + & 32.8 & $0 / 20$ & - & - \\
\hline $\mathrm{X}$ & 28.3 & & & 35.9 & & & \\
\hline RANGO & $16-43$ & & & $26.6-45.6$ & & & \\
\hline SEM & \pm 2.1 & & & \pm 1.6 & & & \\
\hline
\end{tabular}

* Antecedentes bilaterales de DM II.

Tabla 1d

PRESENTACION CLINICA DE LAS MUJERES DEL GRUPO IV $n=15$ NO OBESAS HIPERANDROGENICAS (A)

\begin{tabular}{|c|c|c|c|c|c|c|c|}
\hline PTE.No. & EDAD A & $\begin{array}{l}\text { ANTEC. } \\
\text { DM II }\end{array}$ & $\begin{array}{l}\text { OLIGOAM. } 0 \\
\text { AMENORREA }\end{array}$ & IMC & IH & $\overline{A N}$ & $\overline{\text { ACNE }}$ \\
\hline 1 & 26 & + & + & 23.4 & $12 / 20$ & + & + \\
\hline 2 & 25 & + & + & 23.8 & $14 / 20$ & + & + \\
\hline 3 & 22 & + & + & 22.6 & $16 / 20$ & + & + \\
\hline 4 & 22 & - & + & 24.3 & $11 / 20$ & - & + \\
\hline 5 & 25 & + & + & 24.3 & $20 / 20$ & - & + \\
\hline 6 & 24 & + & + & 23.1 & $10 / 20$ & - & + \\
\hline 7 & 24 & + & + & 21.4 & $11 / 20$ & - & + \\
\hline 8 & 21 & - & + & 22.0 & $15 / 20$ & - & - \\
\hline 9 & 20 & + & + & 23.3 & $10 / 20$ & - & + \\
\hline 10 & 20 & + & - & 21.9 & $9 / 20$ & - & + \\
\hline 11 & 32 & + & + & 23.6 & $10 / 20$ & - & + \\
\hline 12 & 19 & $+*$ & - & 22.4 & $6 / 20$ & - & + \\
\hline 13 & 28 & + & + & 22.0 & $20 / 20$ & - & + \\
\hline 14 & 26 & + & + & 22.7 & $13 / 20$ & - & + \\
\hline 15 & 30 & + & + & 21.6 & $6 / 20$ & - & + \\
\hline $\mathrm{X}$ & 24.2 & & & 22.8 & & & \\
\hline RANGO & $19-32$ & & & $21.4-24.3$ & & & \\
\hline SEM & \pm 0.9 & & & \pm 0.2 & & & \\
\hline
\end{tabular}

* Antecedentes bilaterales de DM II 
Tabla 1e

HALLAZGOS CLINICOS EN LOS CUATRO GRUPOS DE MUJERES

\begin{tabular}{|c|c|c|c|c|c|c|c|}
\hline GRUPO & \multicolumn{3}{|c|}{$\begin{array}{cc}\text { EDAD ANTEC. } & \text { OLIGOAM. O } \\
\text { DM II } & \text { AMENORREA } \\
\% & \%\end{array}$} & IMC & IH & $\begin{array}{l}\text { AN } \\
\%\end{array}$ & $\begin{array}{c}\text { ACNE } \\
\%\end{array}$ \\
\hline & 23.8 & & 20.5 & & & & \\
\hline I & $\begin{array}{r}20-28 \\
\pm 0.7 \\
28.2\end{array}$ & 33 & $\begin{array}{c}0 \\
\pm 0.4 \\
30.5\end{array}$ & $18.5-24$ & $0 / 20$ & 0 & 0 \\
\hline II & $\begin{array}{r}18-38 \\
\pm 1.2 \\
28.3\end{array}$ & 70 & $\begin{array}{c}80 \\
\pm 1.0 \\
35.9\end{array}$ & $25.3-41.3$ & $9.4 / 20$ & 45 & 55 \\
\hline III & $\begin{array}{r}16-43 \\
\pm 2.1 \\
24.2\end{array}$ & 80 & $\begin{array}{c}20 \\
\pm 1.6 \\
22.8\end{array}$ & $26.6-45.6$ & $0 / 20$ & 33 & 0 \\
\hline IV & $\begin{array}{r}19-32 \\
\pm 0.9\end{array}$ & 86.7 & $\begin{array}{l}86.7 \\
\pm 0.2\end{array}$ & $21.4-24.3$ & $12 / 20$ & 20 & 93.3 \\
\hline
\end{tabular}

estándar del promedio (SEM); las diferencias entre los grupos se establecen mediante análisis de varianza (ANOVA), y su significación estadística se establece con el método de DUNCAN para comparación de varios promedios, en el cual a los promedios estadísticamente diferentes se les asignan letras diferentes. Se consideran niveles altamente significativos cuando $\mathrm{p}<0.001(* *)$, y débilmente significativos cuando $\mathrm{p}<0.05(*)$.

\section{Resultados}

Las características clínicas de las mujeres de cada uno de los grupos estudiados, aparecen en las Tablas 1a, 1b, 1c y ld y un resumen de los datos obtenidos se muestra en la Tabla le.

En ontramos que las edades son similares en los cuatro grupos de pacientes. Nos llamó la atención el hecho de encontrar con mayor frecuencia antecedentes familiares de diabetes mellitus tipo II, en los tres grupos de pacientes (OA) Grupo II, (O) Grupo III y (A) Grupo IV; 70\%, 80\% y $86.7 \%$ respectivamente, en comparación con el $33 \%$ encontrado en el grupo control (C) Grupo I. Tabla le.

Las alteraciones menstruales anteriormente descritas, fueron el hallazgo característico en las mujeres hiperandrogénicas, observándose en el $80 \%$ de las obesas y en el $86.7 \%$ de las delgadas; sin embargo, observamos también estas alteraciones en el $20 \%$ de las mujeres obesas no hiperandrogénicas $(\mathrm{O})$ Grupo III. Tabla le.

El índice de hirsutismo, así como la presencia de acné, fueron de mayor severidad en las mujeres delgadas hiperandrogénicas (A) Grupo IV, que en las obesas hiperandrogénicas (OA) Grupo II (12.2/20 vs. $9.4 / 20)$ y $(93.3 \%$ vs. $55 \%)$, respectivamente. Tabla le.

La Acantosis Nigricans, alteración dermatológica asociada a resistencia a la insulina, se presentó en 9 de las 20 pacientes (OA) Grupo II (45\%), en 5 de las 15 pacientes (O) Grupo III (33\%) y en 3 de las 15 pacientes (A) Grupo IV $(20 \%)$, a pesar de ser estas últimas de peso normal.

Los resultados de las curvas de glicemia e insulinemia de cada una de las mujeres de los diferentes grupos, se presentan en las Tablas $2 \mathrm{a}, 2 \mathrm{c}$ y $2 \mathrm{~d}$, y los promedios de cada grupo + SEM se muestran en la Tabla $2 \mathrm{e}$ y en los Gráficos 1 y 2 .

Los resultados más interesantes los observamos al comparar los promedios de los diferentes grupos:
La dispersión de los datos obtenidos en nuestro grupo control (C) Grupo I en cuanto a glicemia, insulinemia, TT y TL, fue muy estrecha, lo que nos permite establecer parámetros propios de normalidad.

El área bajo la curva de glicemia de las mujeres obesas hiperandrogénicas resultó estadísticamente diferente de la obtenida en el grupo control (C) Grupo I $(341+27.5$ vs. $233+6.6$ ); y de la encontrada en el grupo de mujeres delgadas hiperandrogénicas (A) Grupo IV (341 + 27.5 vs. $254+13.3$ ); sin embargo, no fue diferente de la encontrada en las mujeres obesas no hiperandrogénicas (O) Grupo III. Tabla 3 y Gráfico 3 .

El estudio del área bajo la curva de insulinemia, mostró que las diferencias encontradas entre el grupo control (C) I y los otros tres grupos (OA) II, (O) III y (A) IV, son altamente significativas; $p \quad 0.001$. También fue estadísticamente diferente el área bajo la curva de insulina de los dos grupos de obesas (OA) II y (O) III, del área obtenida en las mujeres hiperandrogénicas de peso normal (A) IV. Tabla 4 y Gráfico 4.

La comparación de los promedios obtenidos en los andrógenos examinados se muestra en las Tablas Nos. 5 y 6 .

Tabla 2a

CURVAS DE GLICEMIA E INSULINA EN LAS MUJERES DEL GRUPO I (C)

\begin{tabular}{|crrrrrr|}
\hline & \multicolumn{7}{c|}{ CURVA DE GLICEMIA } \\
Pte. No. & $\mathbf{0}^{\prime}$ & $\mathbf{3 0}^{\prime}$ & $\mathbf{6 0}$ & $\mathbf{1 2 0 ^ { \prime }}$ & $\mathbf{1 8 0 ^ { \prime }}$ & Area \\
\hline 1 & 72 & 104 & 79 & 63 & 60 & 222 \\
2 & 76 & 102 & 99 & 94 & 53 & 265 \\
3 & 75 & 101 & 66 & 60 & 52 & 205 \\
4 & 71 & 110 & 83 & 86 & 65 & 253 \\
5 & 74 & 106 & 86 & 80 & 80 & 256 \\
6 & 76 & 103 & 79 & 74 & 64 & 236 \\
7 & 74 & 91 & 68 & 77 & 74 & 229 \\
8 & 85 & 134 & 88 & 81 & 72 & 271 \\
9 & 80 & 91 & 91 & 79 & 52 & 239 \\
10 & 81 & 101 & 91 & 81 & 75 & 217 \\
11 & 77 & 108 & 72 & 66 & 66 & 226 \\
12 & 77 & 118 & 83 & 83 & 76 & 261 \\
13 & 77 & 116 & 71 & 71 & 62 & 232 \\
14 & 74 & 93 & 71 & 67 & 64 & 217 \\
15 & 72 & 66 & 64 & 52 & 43 & 173 \\
\hline X & 76 & 103 & 79.4 & 74.2 & 63.8 & 233 \\
ES & \pm 0.9 & \pm 3.9 & \pm 2.7 & \pm 2.8 & \pm 2.7 & \pm 6.6 \\
\hline
\end{tabular}

\begin{tabular}{|c|c|c|c|c|c|c|}
\hline \multirow[b]{2}{*}{ Pte. No. } & \multicolumn{5}{|c|}{ CURVA DE INSULINA } & \multirow[b]{2}{*}{ Area } \\
\hline & $0^{\prime}$ & $30^{\prime}$ & $60^{\prime}$ & $120^{\prime}$ & $180^{\prime}$ & \\
\hline 1 & 9.5 & 105 & 60 & 46 & 6.5 & 149 \\
\hline 2 & 12.0 & 50 & 28 & 23 & 21.0 & 83 \\
\hline 3 & 11.0 & 65 & 28 & 23 & 12.0 & 85 \\
\hline 4 & 13.5 & 55 & 50 & 21 & 16.0 & 97 \\
\hline 5 & 13.5 & 100 & 85 & 36 & 16.0 & 161 \\
\hline 6 & 9.5 & 63 & 41 & 25 & 13.0 & 96 \\
\hline 7 & 6.0 & 120 & 50 & 17 & 11.5 & 121 \\
\hline 8 & 13.0 & 100 & 44 & 25 & 16.5 & 92 \\
\hline 9 & 7.0 & 25 & 21 & 20 & 6.0 & 53 \\
\hline 10 & 5.0 & 16 & 10 & 23 & 17.0 & 48 \\
\hline 11 & 15.5 & 65 & 44 & 29 & 17.0 & 107 \\
\hline 12 & 8.0 & 46 & 32 & 28 & 15.0 & 85 \\
\hline 13 & 5.5 & 32 & 32 & 15 & 5.0 & 59 \\
\hline 14 & 5.0 & 28 & 46 & 25 & 7.0 & 78 \\
\hline 15 & 13.0 & 75 & 37 & 20 & 13.0 & 126 \\
\hline$X$ & 9.8 & 63 & 40.5 & 25 & 12.8 & 96 \\
\hline ES & \pm 0.9 & \pm 8.2 & \pm 4.5 & \pm 2.0 & \pm 1.2 & \pm 8.4 \\
\hline
\end{tabular}


Tabla $2 b$

CURVAS DE GLICEMIA E INSULINA

EN LAS MUJERES DEL GRUPO II (OA)

\begin{tabular}{|c|c|c|c|c|c|c|}
\hline \multirow[b]{2}{*}{ Pte. No. } & \multicolumn{5}{|c|}{ CURVA DE GLICEMIA } & \multirow[b]{2}{*}{ Area } \\
\hline & $0^{\prime}$ & $30^{\prime}$ & $60^{\prime}$ & $120^{\prime}$ & $180^{\prime}$ & \\
\hline 1 & 82 & 107 & 142 & 90 & 65 & 303 \\
\hline 2 & 100 & 114 & 85 & 114 & 88 & 304 \\
\hline 3 & 95 & 192 & 203 & 209 & 100 & 531 \\
\hline 4 & 75 & 115 & 77 & 77 & 71 & 247 \\
\hline 5 & 100 & 170 & 191 & 156 & 63 & 440 \\
\hline 6 & 91 & 157 & 123 & 69 & 48 & 287 \\
\hline 7 & 82 & 124 & 98 & 68 & 53 & 251 \\
\hline 8 & 73 & 104 & 118 & 79 & 64 & 270 \\
\hline 9 & 129 & 249 & 260 & 275 & 213 & 733 \\
\hline 10 & 96 & 128 & 65 & 96 & 75 & 270 \\
\hline 11 & 89 & 124 & 120 & 103 & 89 & 266 \\
\hline 12 & 85 & 150 & 120 & 100 & 59 & 316 \\
\hline 13 & 120 & 210 & 225 & 151 & 102 & 506 \\
\hline 14 & 85 & 134 & 142 & 119 & 104 & 366 \\
\hline 15 & 93 & 123 & 111 & 89 & 82 & 298 \\
\hline 16 & 88 & 160 & 106 & 85 & 32 & 283 \\
\hline 17 & 73 & 117 & 110 & 104 & 97 & 312 \\
\hline 18 & 82 & 148 & 69 & 82 & 59 & 258 \\
\hline 19 & 69 & 148 & 119 & 104 & 100 & 334 \\
\hline 20 & 86 & 119 & 96 & 70 & 45 & 245 \\
\hline $\mathrm{x}$ & 89.6 & 144.6 & 129 & 112 & 80.4 & 341 \\
\hline ES & \pm 3.36 & \pm 8.4 & \pm 11.8 & \pm 11.7 & \pm 8.5 & \pm 27.5 \\
\hline \multicolumn{7}{|c|}{ CURVA DE INSULINA } \\
\hline Pte. No. & $\mathbf{0}^{\prime}$ & $30^{\prime}$ & $60^{\prime}$ & $120^{\prime}$ & $180^{\prime}$ & Area \\
\hline 1 & 12.5 & 145 & 120 & 110 & 65 & 308 \\
\hline 2 & 14.0 & 280 & 105 & 215 & 145 & 512 \\
\hline 3 & 22.0 & 150 & 150 & 180 & 38 & 391 \\
\hline 4 & 14.0 & 400 & 110 & 60 & 45 & 394 \\
\hline 5 & 18.0 & 80 & 80 & 75 & 42 & 201 \\
\hline 6 & 62.0 & 250 & 140 & 90 & 85 & 378 \\
\hline 7 & 25.0 & 260 & 270 & 200 & 32 & 555 \\
\hline 8 & 16.5 & 400 & 350 & 290 & 190 & 852 \\
\hline 9 & 82.0 & 388 & 346 & 400 & 400 & 1074 \\
\hline 10 & 13.0 & 190 & 190 & 160 & 85 & 443 \\
\hline 11 & 17.5 & 290 & 190 & 110 & 40 & 442 \\
\hline 12 & 11.5 & 75 & 80 & 40 & 9.5 & 145 \\
\hline 13 & 48.0 & 230 & 350 & 350 & 150 & 815 \\
\hline 14 & 16.0 & 155 & 400 & 200 & 85 & 624 \\
\hline 15 & 24.0 & 180 & 130 & 160 & 105 & 406 \\
\hline 16 & 14.5 & 220 & 220 & 130 & 20 & 419 \\
\hline 17 & 10.0 & 150 & 130 & 90 & 65 & 298 \\
\hline 18 & 10.0 & 180 & 130 & 68 & 15 & 266 \\
\hline 19 & 25.0 & 200 & 400 & 210 & 270 & 751 \\
\hline 20 & 9.5 & 20 & 105 & 80 & 15 & 269 \\
\hline $\mathrm{X}$ & 23.2 & 221 & 199.8 & 160.9 & 95 & 476.2 \\
\hline ES & \pm 4.3 & \pm 21.4 & \pm 25.0 & \pm 22.2 & \pm 22.2 & \pm 53.4 \\
\hline
\end{tabular}

La TT en las mujeres obesas hiperandrogénicas (DA) Grupo II, fue estadísticamente diferente de la obtenida en los otros tres grupos; $\mathrm{p} \quad 0.05$.

En cuanto a la TL, los niveles obtenidos en los grupos de mujeres hiperandrogénicas (OA) II y (A) IV, fueron significativamente diferentes de los encontrados en las mujeres que no presentaban signos de androgenización (C) I y (O) III; p 0.05 .

En razón de la alta frecuencia de antecedentes familiares de diabetes mellitus tipo II en nuestros tres grupos de pacientes (OA) II, (O) III y (A) IV, pretendimos establecer si aquellas mujeres con historia familiar positiva para esta patología, tienen diferencias en su glicemia e insulinemia en respuesta a la carga oral de glucosa. En cuanto al área bajo la curva de glicemia no existen diferencias cuando hay antecedentes, ya sea en uno o en ambos padres, o cuando no los hay. Por el contrario, existe una diferencia significativa entre el área bajo la curva de insulina de las mujeres con antecedente familiar bilateral de diabetes mellitus tipo II, p

0.05 ; pero no cuando este antecedente compromete a uno solo de los padres. Tabla 7.

La Acantosis Nigricans es un invariable marcador de resistencia a la insulina e hiperinsulinemia. Las diferencias que observamos, tanto en la insulina basal como en el área bajo la curva de insulinemia, fueron estadísticamente significativas, $\mathrm{p} \quad 0.001$, cuando se compararon las mujeres que exhibían esta anomalía dermatológica con las que no la presentaban. Tabla 8 y Gráfico 5 .

\section{Discusión}

El papel etiológico que tiene la hiperinsulinemia, generalmente consecuencia de una resistencia periférica a la insulina, en la hipertensión arterial (10), el infarto del miocardio (11) y la hiperuricemia (12), es un hecho de considerable importancia en la comprensión del origen de entidades, de

Tabla 2c

CURVAS DE GLICEMIA E INSULINA

EN LAS MUJERES DEL GRUPO III (O)

\begin{tabular}{|crrrrrr|}
\hline Pte. No. & $\mathbf{0}^{\prime}$ & $\mathbf{3 0}^{\prime}$ & $\mathbf{6 0}^{\prime}$ & $\mathbf{1 2 0}^{\prime}$ & $\mathbf{1 8 0}^{\prime}$ & Area \\
\hline 1 & 117 & 169 & 185 & 117 & 74 & 407 \\
2 & 85 & 138 & 104 & 108 & 100 & 325 \\
3 & 82 & 97 & 79 & 86 & 55 & 242 \\
4 & 77 & 122 & 137 & 150 & 101 & 383 \\
5 & 89 & 157 & 135 & 89 & 86 & 334 \\
6 & 82 & 107 & 55 & 82 & 82 & 338 \\
7 & 66 & 117 & 93 & 53 & 53 & 224 \\
8 & 93 & 159 & 133 & 110 & 110 & 368 \\
9 & 81 & 130 & 78 & 111 & 47 & 278 \\
10 & 79 & 104 & 79 & 82 & 66 & 246 \\
11 & 76 & 111 & 64 & 55 & 40 & 197 \\
12 & 86 & 168 & 157 & 119 & 45 & 365 \\
13 & 77 & 139 & 100 & 57 & 42 & 242 \\
14 & 78 & 119 & 81 & 94 & 61 & 264 \\
15 & 78 & 78 & 75 & 66 & 54 & 208 \\
\hline$X$ & 83.1 & 127 & 103.6 & 91.9 & 67.6 & 288 \\
ES & \pm 2.9 & \pm 6.9 & \pm 9.7 & \pm 7.1 & \pm 6.2 & \pm 17.8 \\
\hline
\end{tabular}

\begin{tabular}{|c|c|c|c|c|c|c|}
\hline \multirow[b]{2}{*}{ Pte. No. } & \multicolumn{5}{|c|}{ CURVA DE INSULINA } & \multirow[b]{2}{*}{ Area } \\
\hline & $\mathbf{0}^{\prime}$ & $30^{\prime}$ & $60^{\prime}$ & $120^{\prime}$ & $180^{\prime}$ & \\
\hline 1 & 17.0 & 400 & 400 & 180 & 110 & 739 \\
\hline 2 & 20.0 & 400 & 270 & 150 & 60 & 585 \\
\hline 3 & 17.5 & 400 & 250 & 170 & 60 & 592 \\
\hline 4 & 50.0 & 400 & 400 & 400 & 400 & 1113 \\
\hline 5 & 30.0 & 400 & 240 & 160 & 110 & 603 \\
\hline 6 & 9.6 & 120 & 140 & 65 & 52 & 258 \\
\hline 7 & 11.5 & 80 & 110 & 120 & 65 & 278 \\
\hline 8 & 24.0 & 200 & 100 & 40 & 40 & 241 \\
\hline 9 & 17.5 & 350 & 220 & 48 & 44 & 441 \\
\hline 10 & 24.0 & 270 & 200 & 160 & 150 & 526 \\
\hline 11 & 25.0 & 310 & 100 & 19 & 10 & 260 \\
\hline 12 & 13.0 & 110 & 180 & 150 & 60 & 373 \\
\hline 13 & 14.0 & 180 & 105 & 85 & 14 & 264 \\
\hline 14 & 15.5 & 235 & 190 & 50 & 11 & 319 \\
\hline 15 & 4.5 & 200 & 95 & 50 & 27 & 236 \\
\hline $\mathrm{X}$ & 19.5 & 270.3 & 200 & 123.1 & 80.9 & 453 \\
\hline ES & \pm 2.76 & & \pm 26 & \pm 24.4 & \pm 25 & \pm 63.3 \\
\hline
\end{tabular}


Tabla 2d

CURVAS DE GLICEMIA E INSULINA EN LAS MUJERES DEL GRUPO IV (A)

\begin{tabular}{|crrrrrr|}
\hline & \multicolumn{7}{c|}{ CURVA DE GLICEMIA } \\
Pte. No. & $\mathbf{0}^{\prime}$ & $\mathbf{3 0}^{\prime}$ & $\mathbf{6 0}$ & $\mathbf{1 2 0 ^ { \prime }}$ & $\mathbf{1 8 0}$ & Area \\
\hline 1 & 90 & 126 & 84 & 87 & 81 & 276 \\
2 & 63 & 79 & 98 & 65 & 70 & 229 \\
3 & 85 & 108 & 72 & 85 & 63 & 246 \\
4 & 78 & 104 & 88 & 88 & 69 & 260 \\
5 & 114 & 181 & 114 & 129 & 105 & 386 \\
6 & 80 & 111 & 71 & 80 & 53 & 235 \\
7 & 67 & 103 & 58 & 62 & 39 & 193 \\
8 & 73 & 107 & 104 & 100 & 61 & 280 \\
9 & 86 & 132 & 100 & 100 & 64 & 295 \\
10 & 87 & 150 & 90 & 83 & 62 & 278 \\
11 & 66 & 69 & 45 & 53 & 42 & 160 \\
12 & 73 & 107 & 93 & 52 & 40 & 214 \\
13 & 77 & 111 & 76 & 89 & 52 & 247 \\
14 & 83 & 99 & 96 & 93 & 93 & 282 \\
15 & 77 & 113 & 83 & 77 & 44 & 237 \\
\hline$X$ & 79.8 & 113.3 & 84.2 & 82.9 & 62.5 & 254.5 \\
ES & \pm 3.17 & \pm 6.9 & \pm 4.25 & \pm 5.1 & \pm 4.9 & \pm 13.3 \\
\hline
\end{tabular}

\begin{tabular}{|c|c|c|c|c|c|c|}
\hline \multirow[b]{2}{*}{ Pte. No. } & \multicolumn{5}{|c|}{ CURVA DE INSULINA } & \multirow[b]{2}{*}{ Area } \\
\hline & $0^{\prime}$ & $30^{\prime}$. & $60^{\prime}$ & $120^{\prime}$ & $180^{\prime}$ & \\
\hline 1 & 16.0 & $\overline{95}$ & 65 & 55 & 52 & 181 \\
\hline 2 & 8.5 & 110 & 250 & 200 & 160 & 525 \\
\hline 3 & 7.0 & 190 & 50 & 60 & 10 & 199 \\
\hline 4 & 8.0 & 100 & 50 & 145 & 60 & 265 \\
\hline 5 & 7.5 & 150 & 140 & 170 & 58 & 381 \\
\hline 6 & 8.0 & 120 & 95 & 46 & 13 & 186 \\
\hline 7. & 16.0 & 230 & 130 & 125 & 14 & 349 \\
\hline 8 & 8.0 & 135 & 110 & 120 & 120 & 332 \\
\hline 9 & 4.4 & 120 & 75 & 100 & 28 & 231 \\
\hline 10 & 22.0 & 100 & 75 & 35 & 18 & 156 \\
\hline 11 & 18.0 & 350 & 275 & 75 & 36 & 479 \\
\hline 12 & 6.5 & 250 & 95 & 36 & 10 & 239 \\
\hline 13 & 15.0 & 300 & 120 & 50 & 25 & 306 \\
\hline 14 & 15.0 & 50 & 36 & 23 & 17.5 & 87 \\
\hline 15 & 11.0 & 62 & 125 & 16 & 5 & 46 \\
\hline$X$ & 11.3 & 157 & 112.7 & 83.7 & 41.7 & 270.8 \\
\hline ES & \pm 1.34 & \pm 22.7 & \pm 17.7 & \pm 14.6 & \pm 11.4 & \pm 32.0 \\
\hline
\end{tabular}

Tabla 2e

PROMEDIO MAS O MENOS SEM

DE LAS CURVAS DE GLICEMIA E INSULINA EN LOS 4 GRUPOS DE MUJERES

\begin{tabular}{|c|c|c|c|c|c|c|}
\hline & \multicolumn{5}{|c|}{ GLICEMIA } & \multirow[b]{2}{*}{ Area } \\
\hline & $\mathbf{0}^{\prime}$ & $30^{\prime}$ & $60^{\prime}$ & $120^{\prime}$ & $180^{\prime}$ & \\
\hline \multirow[t]{2}{*}{ I } & 76 & 103 & 79.4 & 74.2 & 63.8 & 233 \\
\hline & \pm 0.9 & \pm 3.9 & \pm 2.7 & \pm 2.8 & \pm 2.7 & \pm 6.6 \\
\hline \multirow[t]{2}{*}{ II } & 89.6 & 144.6 & 129 & 112 & 80.4 & 341 \\
\hline & \pm 3.36 & \pm 8.4 & \pm 11.8 & \pm 11.7 & \pm 8.5 & \pm 27.5 \\
\hline \multirow[t]{2}{*}{ III } & 83.1 & 127 & 103.6 & 91.9 & 67.6 & 288 \\
\hline & \pm 2.9 & \pm 6.9 & \pm 9.7 & \pm 7.1 & \pm 6.2 & \pm 17.8 \\
\hline \multirow[t]{2}{*}{ IV } & 79.8 & 113.3 & 84.2 & 82.9 & 62.5 & 254.5 \\
\hline & \pm 3.17 & \pm 6.9 & \pm 4.25 & \pm 5.1 & \pm 4.9 & \pm 13.3 \\
\hline
\end{tabular}

\begin{tabular}{|crrrrrr|}
\hline \multicolumn{7}{c|}{ INSULINEMIA } \\
& $0^{\prime}$ & $30^{\prime}$ & $\mathbf{6 0}$ & $\mathbf{1 2 0}$ & \multicolumn{1}{c|}{$180^{\prime}$} & Area \\
\hline I & 9.8 & 63 & 40.5 & 25 & 12.8 & 96 \\
& \pm 0.9 & \pm 8.2 & \pm 4.5 & \pm 2.0 & \pm 1.2 & \pm 8.4 \\
II & 23.2 & 221 & 199.8 & 160.9 & 95 & 476.2 \\
& \pm 4.3 & \pm 21.4 & \pm 25.0 & \pm 22.2 & \pm 22.2 & \pm 53.4 \\
III & 19.5 & 270.3 & 200 & 123.1 & 80.9 & 453 \\
& \pm 2.76 & \pm 31.2 & \pm 26 & \pm 24.4 & \pm 25 & \pm 63.3 \\
IV & 11.3 & 157 & 112.7 & 83.7 & 41.7 & 270.8 \\
& \pm 1.34 & \pm 22.7 & \pm 17.7 & \pm 14.6 & \pm 11.4 & \pm 32.0 \\
\hline
\end{tabular}

Gráfico 1

CURVAS DE TOLERANCIA A LA GLUCOSA EN LOS 4 GRUPOS DE MUJERES

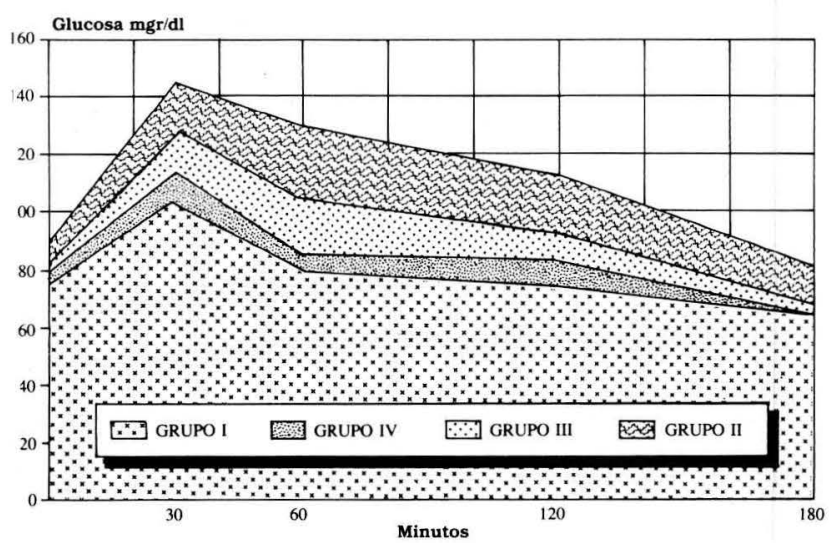

Gráfico 2

CURVAS DE INSULINA

EN LOS 4 GRUPOS DE MUJERES

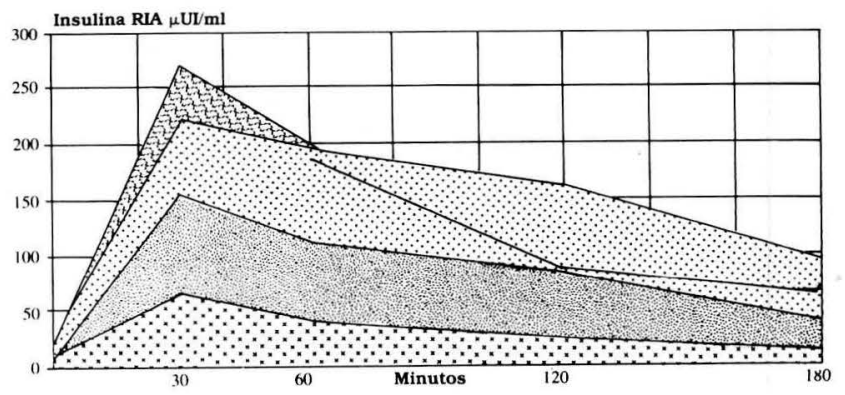

$\because$ GRUPO I GRUPO IV G GRUPO II

Tabla 3

PROMEDIO MAS O MENOS SEM DE LOS DATOS OBTENIDOS EN CADA GRUPO DE PACIENTES

\begin{tabular}{|crrrrrrl|}
\hline GRUPO & EDAD & IMC & AG & IB & AI & TT & TL \\
\hline I & 23.8 & 20.5 & 233.4 & 9.8 & 96.0 & 0.87 & 1.44 \\
& 0.7 & 10.4 & 6.6 & \pm 0.9 & 8.4 & 0.1 & 0.3 \\
II & 28.2 & 30.5 & 341 & 23.2 & 476.2 & 1.24 & 3.28 \\
& 1.2 & 1.0 & 27.5 & \pm 4.3 & 53.4 & 0.1 & 0.3 \\
III & 28.3 & 35.9 & 288 & 19.5 & 453 & 0.91 & 1.78 \\
& 2.1 & 1.6 & 17.8 & \pm 2.7 & 63.3 & 0.09 & 0.2 \\
IV & 24.2 & 22.8 & 254 & 11.3 & 270.8 & 0.83 & 2.94 \\
& 0.9 & 0.2 & 13.3 & \pm 1.3 & 32.0 & 0.09 & 0.4 \\
\hline
\end{tabular}

no poca frecuencia, hasta ahora calificadas como "esenciales" o de origen multifactorial.

En este trabajo nuestro propósito fue dar un paso adelante en el estudio del papel que tiene el hiperinsulinismo en los cuadros de androgenización de la mujer. Un estudio previo nos había mostrado que existe una correlación muy positiva entre el grado de obesidad medido por el IMC y los síntomas o signos de androgenización, en especial las alteraciones menstruales y el hirsutismo (26).

Razonando que la obesidad se acompaña siempre de hiperinsulinemia y que diferentes trabajos encuentran la presencia 
Gráfico 3

PROMEDIO DE AREA BAJO LA CURVA DE GLICEMIA

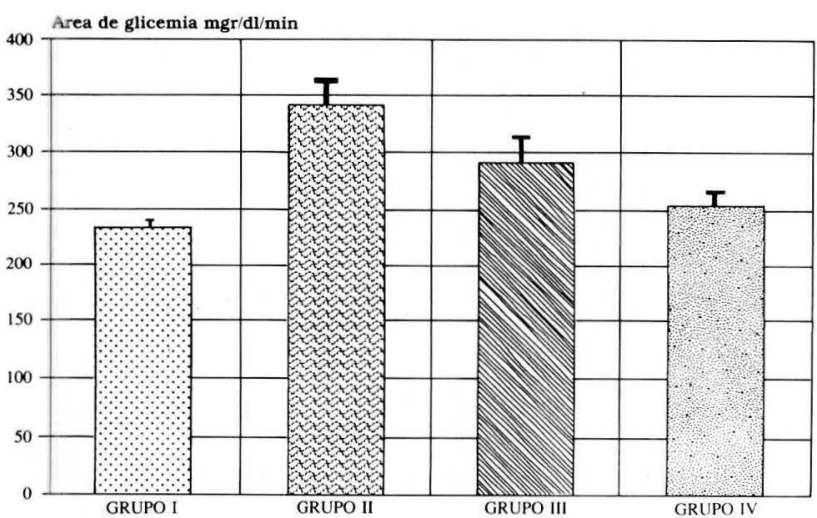

Tabla 4

COMPARACION DE PROMEDIOS DE AREA

BAJO LA CURVA DE GLICEMIA E INSULINEMIA

EN LOS CUATRO GRUPOS. APLICACION DEL METODO DE DUNCAN PARA SIGNIFICACION ESTADISTICA

\begin{tabular}{|rrrr|}
\hline GRUPO & No. & AG & DUNCAN \\
I & 15 & 233.4 & B \\
II & 20 & 341.0 & A \\
III & 15 & 288.0 & AB \\
IV & 15 & 254.0 & B \\
\cline { 2 - 4 } & & & p<0.05 \\
\hline GRUPO & No. & AI & DUNCAN \\
I & 15 & 96.0 & C \\
II & 20 & 476.2 & A \\
III & 15 & 453.0 & B \\
IV & 15 & 270.8 & p<0.001 \\
& & & \\
\hline
\end{tabular}

Gráfico 4

PROMEDIO DE AREA BAJO LA CURVA DE INSULINA

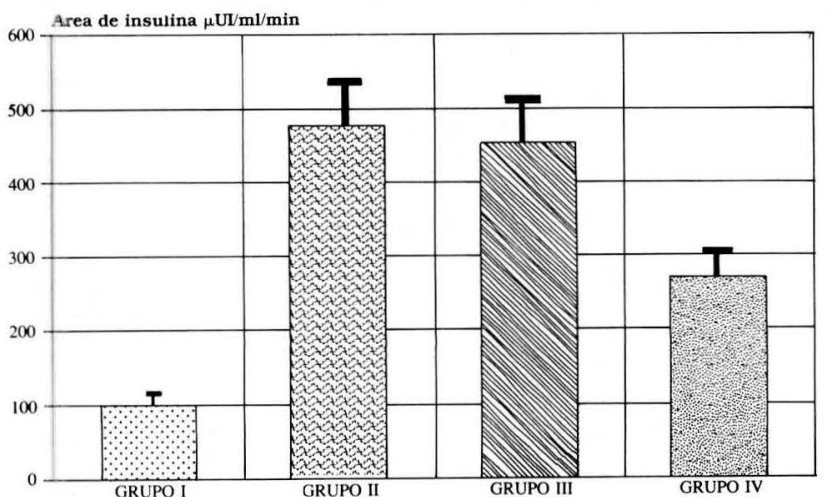

de un exceso de insulina asociado al exceso de producción de andrógenos $(3-9,29)$, parece lógico establecer una relación causal en el sentido hiperinsulinemia $\Rightarrow$ exceso de producción de andrógenos $\Rightarrow$ androgenización.

Sin embargo, la observación frecuente de mujeres obesas sin androgenización, como también de mujeres delgadas con androgenización, plantea el problema de comprender cómo en ciertas mujeres la hiperinsulinemia conduce a obesidad más androgenización, mientras que en otras solamente se manifiesta, ya sea por obesidad, ya sea por androgenización. El diseño de cuatro grupos de mujeres que representaran las características clínicas arriba descritas y la normali-

Tabla 5

COMPARACION DE PROMEDIOS DE TT Y TL EN LOS CUATRO GRUPOS. APLICACION DEL METODO DE DUNCAN PARA SIGNIFICACION ESTADISTICA

\begin{tabular}{|cccc|}
\hline GRUPO & No. & PROM & DUNCAN \\
\hline I & 15 & 0.87 & B \\
II & 20 & 1.24 & A \\
III & 15 & 0.91 & B \\
IV & 15 & 0.83 & B \\
& & & p<0.05 \\
\hline
\end{tabular}

Tabla 6

\begin{tabular}{|cccc|}
\hline GRUPO & No. & PROM. & DUNCAN \\
\hline I & 15 & 1.44 & B \\
II & 20 & 3.28 & A \\
III & 15 & 1.78 & B \\
IV & 15 & 2.94 & A \\
& & & p<0.05 \\
\hline
\end{tabular}

Tabla 7

DIFERENCIAS ENTRE AREA DE GLICEMIA E INSULINA CUANDO HAY HISTORIA FAMILIAR DE DM II

\begin{tabular}{|c|c|c|c|}
\hline \multicolumn{4}{|c|}{ AREA GLICEMIA } \\
\hline $\begin{array}{r}\text { ANTEC } \\
\text { DM II }\end{array}$ & $\mathbf{x}$ & No. & DUNCAN \\
\hline BILAT. & 341.6 & 9 & A \\
\hline UNILAT. & 285.6 & 9 & $\mathrm{AB}$ \\
\hline NEGAT. & 256.6 & 21 & B \\
\hline & & & NS \\
\hline \multicolumn{4}{|c|}{ AREA INSULINA } \\
\hline $\begin{array}{r}\text { ANTEC } \\
\text { DM II }\end{array}$ & $\mathbf{x}$ & No. & DUNCAN \\
\hline BILAT. & 525.7 & 9 & $\mathrm{~A}$ \\
\hline UNILAT. & 342.8 & 35 & B \\
\hline NEGAT. & 223.6 & 21 & B \\
\hline & & & $p<0.05$ \\
\hline
\end{tabular}

Tabla 8

DIFERENCIAS ENTRE INSULINA BASAL Y AREA DE INSULINA CUANDO EXISTE O NO AN

\begin{tabular}{|c|c|c|c|}
\hline \multicolumn{4}{|c|}{ INSULINA BASAL } \\
\hline AN & $\mathbf{x}$ & No. & DUNCAN \\
\hline CONAN & 25.4 & 17 & A \\
\hline SIN AN & 13.4 & 48 & B \\
\hline & & & $p<0.001$ \\
\hline \multicolumn{4}{|c|}{ AREA INSULINA } \\
\hline AN & $\mathbf{x}$ & No. & DUNCAN \\
\hline CON AN & 517.8 & 17 & A \\
\hline SIN AN & 263.0 & 48 & $\mathrm{~B}$ \\
\hline & & & $p<0.001$ \\
\hline
\end{tabular}


Gráfico 5

INSULINA BASAL Y AREA DE INSULINA

EN MUJERES SIN Y CON AN

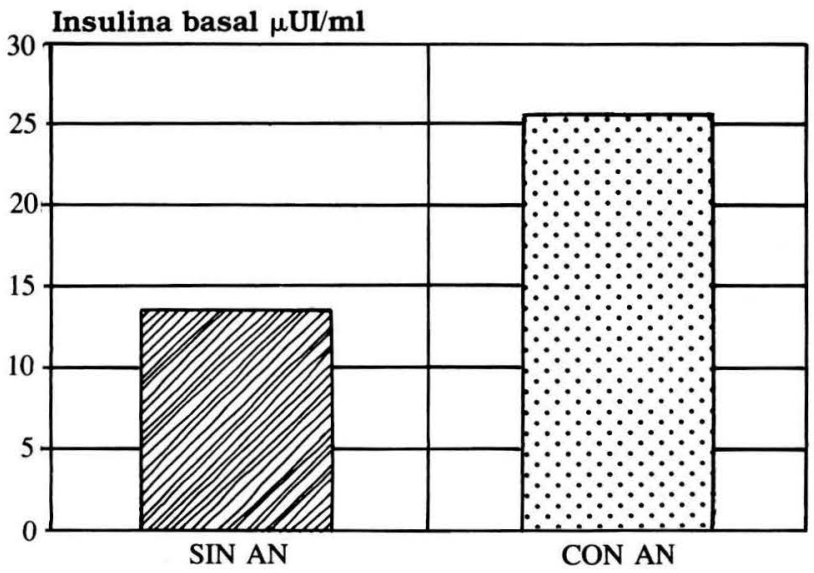

dad, nos permitió comprender los mecanismos responsables de la variación en la presentación de estos fenómenos.

Encontramos en el grupo de mujeres obesas con signos de hiperandrogenismo (OA) Grupo II, los niveles más altos de insulina, en algunos casos con intolerancia a la glucosa, y niveles elevados de andrógenos (TT y TL); la presencia de Acantosis Nigricans en estas mujeres fue un marcador de la severidad de su resistencia a la insulina. Este grupo parece corresponder de una manera muy exacta con la descripción hecha por Stein y Leventhal, del Síndrome de Ovario Poliquístico en 1935 (18); y existen en la literatura amplias descripciones del fenómeno (30-32). En las mujeres de este grupo es indiscutible el efecto causal de la hiperinsulinemia, sobre el exceso de andrógenos encontrado.

En lo que concierne al grupo de mujeres obesas sin signos de androgenización (O) Grupo III, encontramos niveles de insulina estadísticamente iguales a los de las mujeres obesas hiperandrógenicas (OA) Grupo II; sin embargo ellas no presentaban, ni en su examen físico ni en sus laboratorios, evidencia de sobreproducción de andrógenos. Nosotros proponemos, como resultado de la observación de estas mujeres, que ellas presentan un tipo de obesidad diferente de la observada en las mujeres obesas hiperandrogénicas, con una distribución femoral o inferior, llamada por algunos hiperplásica. Es un hecho conocido que la obesidad localizada en la mitad superior del cuerpo, está asociada con severa resistencia a la insulina, intolerancia a carbohidratos y androgenización en la mujer (33-35); podría pensarse que las mujeres con una distribución topográfica de su tejido graso en el segmento inferior, tendrían mayor capacidad de aromatización periférica de andrógenos a estrógenos (36), lo que podría atenuar la elevación y actividad de los primeros. Con este planteamiento Grenman y col. (34), Evans y col. (33) y Dunaif y col. (36), han pretendido establecer si estas mujeres obesas no hiperandrogénicas tienen niveles elevados de estrógenos; sin embargo, ellos no han encontrado diferencias significativas en estas hormonas, cuando comparan sus grupos de obesas no hiperandrogénicas con mujeres de peso normal.

Nosotros hemos intentado, basados en el índice cintura abdominal/cintura pélvica, establecer cuándo la obesidad es

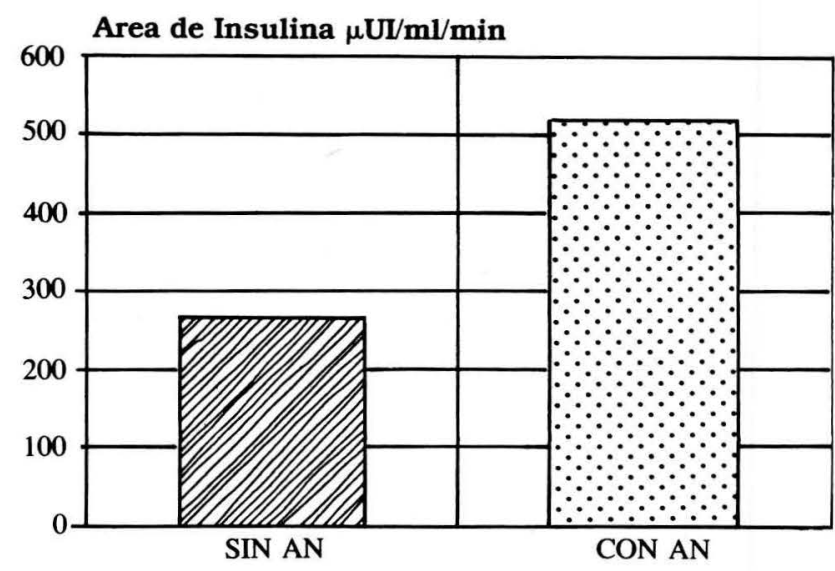

del segmento superior y cuándo del segmento inferior del cuerpo, para establecer si esta distribución topográfica se correlaciona significativamente con los niveles de esteroides sexuales encontrados, lo cual será motivo de posteriores estudios.

De otra parte, nuestro grupo de mujeres hiperandrogénicas de peso normal (A) Grupo IV, resultó con niveles de insulina significativamente mayores que los encontrados en mujeres normales; este hecho nos permite proponer a la hiperinsulinemia como el factor causal en el desarrollo de su androgenización. Lo anterior ha sido puesto en evidencia en estudios de mujeres hirsutas de peso normal publicados por otros autores (5-7). Nosotros no podemos explicar el por qué de la ausencia de obesidad en estas mujeres; posiblemente estén involucrados otros factores, dependientes de la carga genética, ambientales, hábitos dietarios, etc.

Puesto que la resistencia a la insulina y la hiperinsulinemia son hallazgos característicos de las etapas iniciales de la diabetes mellitus tipo II, y dada la importancia de la herencia en la presentación de este fenómeno, nosotros pretendimos establecer si nuestras mujeres tenían en su historia familiar antecedentes de DM tipo II u obesidad; encontramos en los tres grupos de mujeres hiperinsulinémicas mayor frecuencia de historia de obesidad y DM tipo II, 70\%, $80 \%$ y $86.7 \%$, en comparación con los antecedentes de las mujeres del grupo control, 33\%. Aunque la determinación de este fenómeno podría ser discutible, en razón de que los datos fueron obtenidos de las pacientes y no corroborados objetivamente, sí parece existir en estas mujeres una predisposición para sufrir enfermedades relacionadas con el mismo evento fisiopatológico. Encontramos además, curiosamente, que cuando existía herencia bilateral tanto materna como paterna, el grado de resistencia a la insulina era significativamente mayor. Esto ha sido descrito no directamente en relación con las mujeres que padecen el Síndrome de Ovario Poliquístico, pero sí en estudios de población de obesos y diabéticos que se caracterizan por resistencia a la insulina e hiperinsulinemia (37).

Finalmente la Acantosis Nigricans se correlacionó significativamente con el grado de resistencia a la insulina, encontrándose en las mujeres que exhibían esta anomalía dermatológica 
los mayores niveles de insulina, tanto basal como post-estímulo con glucosa. Estos hallazgos se correlacionan con los encontrados por otros autores $(4,8,29)$. Las mujeres hiperandrogénicas con Acantosis Nigricans tuvieron niveles de andrógenos similares a las que no presentaban esta anomalía, sugiriendo que la Acantosis Nigricans constituye un subgrupo de mujeres con un desorden endocrino asociado, que posiblemente involucra más directamente a los receptores para la insulina.

En conclusión, nosotros proponemos que la hiperinsulinemia es el factor causal de anomalías en la función reproductiva de la mujer posiblemente derivadas de una hiperestimulación ovárica sobre la esteroidogénesis que culmina en una mayor producción de andrógenos. Creemos que las diferencias observadas en los niveles de andrógenos y en la presentación clínica son dependientes de la distribución topográfica de la grasa en las mujeres obesas; y estamos convencidos de que una disminución en los niveles de insulina en estas pacientes representa una mejoría en su cuadro clínico, hecho que hemos observado en algunas de nuestras pacientes sometidas a pérdida de peso.

Proponemos el diseño de un estudio comparativo en mujeres hiperinsulinémicas, hiperandrogénicas, antes y después de ser sometidas a un régimen especial de disminución de peso.

\section{BIBLIOGRAFIA}

1. Reyes-Leal B, Ardila E, Carrillo JC. Diabetes mellitus tipo II. Act Med Col 1980; 5: 359:

2. Doeden B, Rizza R. Use of a variable insulin infusion to assess insulin action in obesity: Defects in both the kinetics and amplitude of response. J Clin Endocrinol Metab 1987; 68: 173.

3. Burghen G, Givens J, Kitabchi A. Correlation of hyperandrogenism with hyperinsulinism in polycystic ovarian disease. J Clin Endocrinol Metab 1980; 50: 113.

4. Barbieri R, Ryan K. Hyperandrogenism, insulin resistance, and Acanthosis Nigricans syndrome: A common endocrinopathy with distinct pathophysiologic features. Am J Obstet Gynecol 1983; 147: 90.

5. Chang R, Nakamura R, Judd H, Kaplan S. Insulin resistance in nonobese patients with polycystic ovarian disease. J Clin Endocrinol Metab 1983; 57: 356.

6. Shoupe D, Kumar D, Lobo R. Insulin resistance in polycistic ovary syndrome. Am J Obstet Gynecol 1983; 147: 588.

7. Jialal I, Naiker P, Reddi K et al. Evidence for insulin resistance in nonobese patients with polycystic ovarian disease. J Clin Endocrinol Metab 1987; 64: 1066.

8. Nestler J, Clore J, Strauss J, Blackard W. The effects of hyperinsulinemia on serum testosterone, progesterone, dehydroepiandrosterone sulfate, and cortisol levels in normal women and in a woman with hyperandrogenism, insulin resistance, and Acanthosis Nigricans. J Clin Endocrinol Metab 1987; 64: 180.

9. Dunaif A, Graf M, Mandeli J et al. Characterization of groups of hyperandrogenic woman with Acanthosis Nigricans, impaired glucose tolerance, and/or hyperinsulinemia. J Clin Endocrinol Metab 1987; 65: 499.

10. Ferrannini E, Buzzigoli $G$, Bonadonna $R$ et al. Insulin resistance in essential hypertension. N Engl J Med 1987; 317: 350

11. Reaven G. Rolle of insulin resistance in human desease. Diabetes 1988; 37: 1595 .

12. Modan $\mathbf{M}$, Halkin H, Karasik A, Luskv A. Elevated-serum uric acid-a facet of hyperinsulinemia. Diabetología 1987; 30: 713.

13. Hammond M, Talbert L, Groff T. Hyperandrogenism. Postgrad Med 1986; 79: 107.

14. Rittmaster R, Loriaux L. Hirsutism. Ann Intern Med 1987; 106: 95.

15. Maroulis G. Evaluation of hirsutism and hyperandrogenemia. Fertil Sterily 1981; 36: 273.

16. Greenblatt RB, Mahesh VB, Grambrell RD. The cause and management of hirsutism. The Parthenon Publishing Group; 1987.

17. Achard C, Thiers J. Le Virilisme Pilaire et son Association a L'insufficance Glycolitique (Diabete a femmes de Barbe). Bull Acad Natl Med (París) 1921; 86: 51.

18. Stein IF, Leventhal ML. Amenorrheà associated with bilateral polycystic ovaries. Am J Obstet Gynecol 1935; 29: 181.

19. Waichenberg BL, Achando SS, Okada $\mathrm{H}$ et al. Determination of the source(s) of androgen over production in hirsutism associated with polycystic ovary syndrome by simultaneous adrenal and ovarian venous catheterization. Comparison with the dexamethasone supression test. J Clin Endocrinol Metab 1986; 63: 1204.

20. Poretsky L, Kalin MF. The gonadotropic function of insulin. Endocr Rev 1987; 8: 132.

21. Veldhuis JD, Toaff ME, Strauss JF, Demers LM. Mechanism subser- ving the trophic actions of insulin on ovarian cells. J Clin Invest 1983; 72: 1046.

22. Barbieri RL, Makris A, Randall RW et al. Insulin stimulates androgen accumulation in incubations of ovarian stroma obtained from woman with hyperandrogenism. J Clin Endocrinol Metab 1986; 62: 904.

23. Garzo VG, Dorrington JH. Aromatase activity in human granulosa cells during follicular development and the modulation by follicle-stimulating hormone and insulin. Am J Obstet Gyr 1984; 148: 657.

24. Couch RM, Muller J, Winter JSD. Regulation of the activities of 17-Hydroxylase and 17,20-Desmolase in the human adrenal cortex: Kinetic Analysis and Inhibition by Endogenous Steroids. J Clin Endocrinol Metab 1986; 63: 613.

25. Voutilainen R, Tapanainen J, Chung B et al. Hormonal Regulation of P450sec (20,22-Desmolase) and P450c17 (17 -Hydroxylase/17,20Lyase) incultured human granulosa cells. J Ciin Endocrinol Metab 1986; 63: 202.

26. Mockus I. Niveles séricos de andrógenos en mujeres hirsutas y su relación con sobrepeso corporal, Niveles séricos de TSH y Prolactina. Trabajo de grado. Universidad Nacional de Colombia. Bogotá. 1986.

27. Moncada-Lorenzo E. Familial study of hirsutism. J Clin Endocrinol Metab 1970; 31: 556.

28. Cody RP, Smith JK. Applied statistics and the SAS programming language. North Holland 1987.

29. Thivolet CH, Mauduit G, Canivet B et al. Acanthosis Nigricans, Hyperandrogénie, Insulino-résistance et hyperlipémie mixte. Presse Med 1988; 17: 1355.

30. Gindoff PR, Jewelewicz R. Polycystic ovarian disease. Obstetrics and Gynecology Clinics of North America 1987; 14: 931.

31. Barbieri RL, Hornstein MD. Hyperinsulinemia and ovarian hyperandrogenism. Endocrinology and Metabolism Clinics of North America 1988; 17: 621 .

32. McKenna TJ. Pathogenesis and treatment of polycystic ovary syndrome. N Engl J Med 1988; 318: 558.

33. Evans DJ, Hoffmann RG, Kalkhoff RK, Kissebah AH. Relationship of androgenic activity to body fat topographic, fat cell morphology, and metabolic aberrations in premenopausal women. J Clin Endocrinol Metab 1983; 57: 304.

34. Grenman S, Ronnemaa T, Irjala $\mathrm{K}$ et al. Sex steroid, gonadotropin, cortisol and prolactin levels in healthy, massively obese women: Correlation with abdominal fat cell size and effect of weigth reduction. J Clin Endocrinol Metab 1986; 63: 1257.

35. Armoni M, Rafaeloff $\mathbf{R}$, Barzilai A et al. Sex differences in insulin action on glucose transport and transporters in human omental adipocytes. J Clin Endocrinol Metab 1987; 65: 1241.

36. Dunaif A, Mandeli J, Fluhr H, Dobrjansky A. The impact of obesity and chronic hyperinsulinemia on gonadotropin release and gonadal steroid secretion in the polycystic ovary syndrome. J Clin Endocrinol Metab 1988; 66: 131.

37. Pettitt DJ, Aleck KA, Baird HR et al. Congenital susceptibility to NIDDM. Diabetes 1988; 37: 622.

38. Pascuali $\mathrm{R}$, Atenucci D, Casimirri $\mathrm{F}$ et al. Clinical and hormonal characteristics of obese amenorrheic women before and after weigth lost. J Clin Endocrinol Metab 1989; 68: 173. 